respiratory functions perioperatively. Superior trunk of brachial plexus visualised accurately through ultrasound, steers effortless performance and achieving acceptable block characteristics.

Methods Here we present a case series of 8 patients aged between 40 years to 80 years with significant restrictive, obstructive pulmonary disease and post-covid patients posted for proximal humerus surgeries. The block was performed under ultrasound guidance, 23G Quincke spinal needle, 1:1 local anaesthetic mixture of $2 \%$ Lignocaine+Adrenaline and $0.5 \%$ Bupivacaine with total volume injected being $15 \mathrm{ml}$. The outcomes measured were loss of shoulder abduction, numbness of shoulder and pain relief, numbness of arm and sonographic assessment of diaphragm movement, oxygen saturation perioperatively and closed-circuit measurement of tidal volume generated

Results 7 patients with superior trunk block achieved complete surgical anaesthesia of proximal shoulder. There was solitary use of Dexmedetomidine for patient with severe anxiety. 2 patients required oxygen supplementation throughout the intraoperative period extending to post-operative period as well. Sonographic assessment intraoperatively and post-operatively disclosed adequate diaphragmatic excursion in all patients. Post-operative pain scores revealed satisfactory pain relief.

Conclusions Effective surgical anaesthesia and analgesia can be delivered considerably with Superior trunk block for surgeries of proximal humerus in patients with compromised pulmonary function by sparing phrenic nerve and it is an appropriate substitute for interscalene block

\section{EVALUATION OF THE PERIPHERAL NERVE STIMULATOR USAGE FOR A SUCCESSFUL COSTACLAVICULAR BRACHIAL PLEXUS BLOCK}

S Soylu*, S Şehirlioğlu, D Genç Moralar, Ü Aygen Türkmen. Gaziosmanpasa Training and Research Hospital/Anesthesiology and Reanimation Department, Istanbul, Turkey

\subsection{6/rapm-2021-ESRA.156}

Background and Aims Ultrasound (US) has become standardized during peripheral nerve block (PNB). Peripheral nerve stimulator (PNS) is combined with US to avoid intraneural injection (1). Monitoring opening injection pressure with a sensor could also show intraneural injection (2). Adding PNS to US guidance did not change block success rates for different types of PNBs $(3,4)$. There is no study in the literature that evaluate effectiveness of adding PNS to US for a successful costaclavicular brachial plexus block (ССВРB). In this randomized prospective clinical study US and injection pressure monitor (IPM) guided CCBPB is compared with US, IPM and PNS guided CCBPB.

Methods 60 patients scheduled for orthopedic surgery below the elbow were enrolled in the study. Ultrasound and IPM guided group called USP and US, IPM and PNS guided group called USPN. In both groups $30 \mathrm{ml}$ bupivacaine-lidocaine mixture was injected when injection pressure is less than 15 psi. In Group USPN, local anesthetic was injected after visualizing the disappearance of motor movement under $0.3 \mathrm{~mA}$ on PNS. Motor and sensorial block levels of the radial, ulnar, median and musculocutaneous nerves were measured. Block success rate, block performance time, number of needle passes and complication rates were compared.
Results Block success rate, number of needle passes and complications were similar (table 1). Block performance time was longer in group USPN (figure 1).

Conclusions Peripheral nerve stimulator is not necessary for a successful CCBPB. Using PNS increased block performance time significantly. Ultrasound and IPM are enough for a successful block with shorter procedure time in CCBPB.
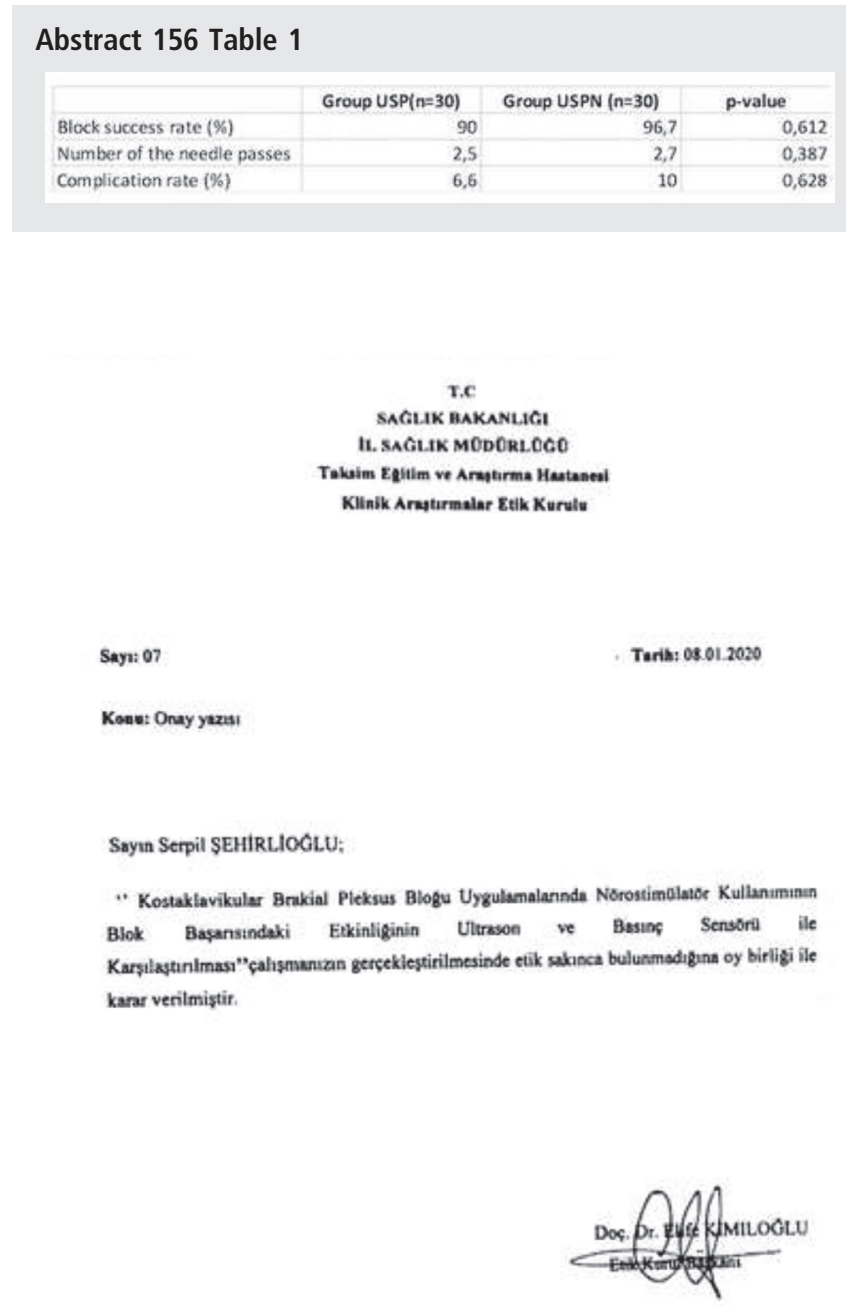

\section{Abstract 156 Figure 1}

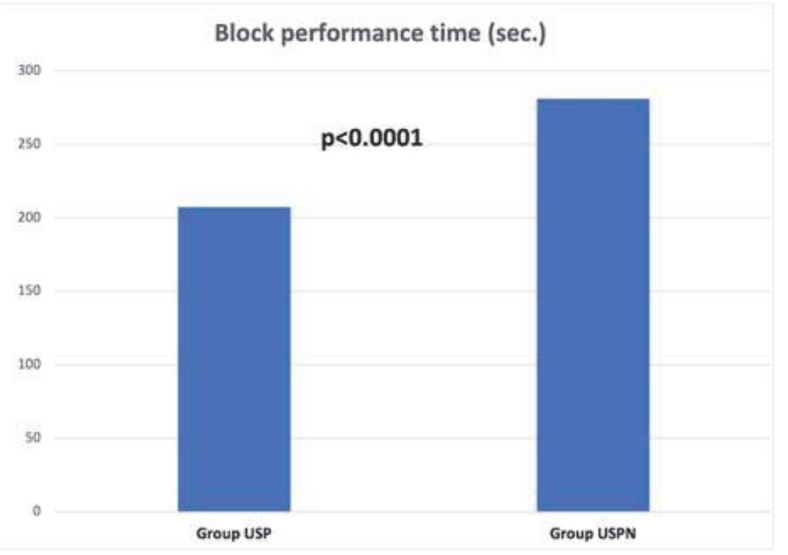

Abstract 156 Figure 2 